Trascender, Contabilidad y Gestión. Vol. 7, Núm. 19 (enero - abril del 2022).

Universidad de Sonora. Departamento de Contabilidad. México.

ISSN: 2448-6388. Reserva de Derechos 04-2015-04172070800-203.

\title{
Identidad universitaria en la comunidad estudiantil de Ciencias Económico Administrativas
}

\author{
University identity in the student community of Economic Administrative Sciences
}

Pablo Alejandro Fava Pedroza ${ }^{1}$; Alma Teresita del Niño Jesús Velarde Mendívil ${ }^{2}$; Lizbeth Salgado Beltrán ${ }^{3}$

Recibido: 10 de agosto de 2021.

Aceptado: 25 de octubre de 2021.

DOI: https://doi.org/10.36791/tcg.v7i19.134

JEL: M31. Marketing.

M32. Imagen.

\section{Resumen}

La identidad visual ayuda a formar una identidad corporativa e imagen de marca sólida, y crea un vínculo con sus mercados de consumo, facilitando analizar las decisiones de compra hacia sus productos. En el terreno educativo los estudiantes al sentir empatía con estos elementos; reflejan pertenencia e identidad hacia su institución. Esta investigación tiene el propósito de describir el grado de sentido de pertenencia e identidad universitaria y visual de los alumnos; y si mantiene sinergia en el nivel de participación de compra hacia los souvenirs universitarios. Se aplica investigación con enfoque cuantitativo, con análisis descriptivo. Se aplicó encuesta a 112 estudiantes de licenciatura cursando últimos semestres de la División de Ciencias Económico y Administrativas. Los resultados muestran un nivel "moderado" de sentido de pertenencia y de identidad universitaria en los

1 Pablo Alejandro Fava Pedroza. Maestro en Administración. Licenciado en Mercadotecnia. Auxiliar de Mercadotecnia del área de Promoción Financiera de la Universidad de Sonora, Hermosillo, Sonora, México. Correo: pablo.fava@unison.mx. ORCID: https://orcid.org/0000-0002-1360-5012.

${ }^{2}$ Alma Teresita del Niño Jesús Velarde Mendívil. Doctora en Dirección y Mercadotecnia. Profesora e Investigadora del Departamento de Contabilidad, Universidad de Sonora, Hermosillo, Sonora, México. Correo: alma.velarde@unison.mx. ORCID https://orcid.org/0000-0003-41646433.

${ }^{3}$ Lizbeth Salgado Beltrán. Doctora en Estudios Empresariales. Profesora e Investigadora del Departamento de Contabilidad, Universidad de Sonora, Hermosillo Sonora, México. Correo electrónico institucional: lizbeth.salgado@unison.mx. ORCID: https://orcid.org/0000-0001-8846275X. 
estudiantes, mientras que en identidad visual señala una evaluación "alta", sin embargo, esto no refleja una relación positiva en comportamiento de consumo de souvenirs universitarios

Palabras clave: identidad, pertenencia, marca.

\begin{abstract}
The visual identity helps to build a corporate identity and strong branding, and creates a link with their consumer's markets, making it easier to analyze the purchasing decisions towards their products. In the field of education to the students feel empathy with these elements; they reflect belonging and identity to their institution. This research has the purpose of describing the degree of sense of belonging and university and visual identity of the students; and if it maintains synergy in the level of purchase participation towards university souvenirs. This research is framed in the scientific methodology, with quantitative approach, under non-experimental design with descriptive analysis. 112 surveys were applied to university students in the last semesters of the Division of Economic and Administrative Sciences. The results show in the students a sense of belonging and university identity at a "moderate" level, while in visual identity it indicates a "high" evaluation, however, this does not reflect a positive relationship in the consumer behavior toward university souvenirs.
\end{abstract}

Keywords: identity, belonging, brand.

\section{Introducción}

El tema de identidad en las universidades abarca una visión más extensa de lo que acontece al interior de los recintos educativos de nivel superior. Experimentar el sentimiento de "formar parte”, es una razón personal y primordial para que los estudiantes puedan alcanzarla; permitiendo así a la institución fortalecer la permanencia de los estudiantes, con la posibilidad de evitar deserciones. El cúmulo de conocimientos que se adquieren en esta etapa van desde valores, pautas conductuales, cooperación, entre otros (Puga, Peschard y Castro, 2007). A través de los años el sentir de los jóvenes estudiantes ha cambiado. De acuerdo con Castro (2015), actualmente, los estudiantes no muestran interés ni estima a los acontecimientos históricos del mundo que los rodea, ocasionando desconocimiento del mismo y una falta de sentimiento y orgullo de formar parte de una entidad. No obstante, es viable poder alcanzar una comunicación e integración estable de la cultura universitaria, por medio de fortalecer a la institucional en colaboración con las autoridades escolares. Múltiples investigaciones, entre ellas la de Osterman (2000), indican un fenómeno en los recintos de educación superior donde se concede prioridad al crecimiento del nivel escolar por encima de las necesidades afectivas de los alumnos, lo que propicia el individualismo y la competencia, dejando los ambientes de colaboración y unión con menor relevancia.

Los estudios de investigación sobre la identidad universitaria e identidad visual en las instituciones educativas son de suma relevancia, ya que favorece, no solo a las instituciones mismas, sino también a su mercado principal: los estudiantes; ya que los diversos grupos estudiantiles de hoy en día se encuentran escasos de métodos que estimulen la motivación e identificación hacia algo, ya que poseen maneras distintas de percibir el entorno (Lobo, 2016).

La comprensión y análisis del nivel de identidad que poseen los estudiantes tiene un alto grado de importancia para las instituciones de educación superior, 
debido a su contribución en el desarrollo de estrategias, encaminadas a generar una mayor identificación de la comunidad estudiantil hacia su universidad, y beneficia a crear procesos que promuevan el crecimiento institucional hacia dentro y fuera de la misma (Cabral, 2006). A través del tiempo, los sucesos habituales del estilo de vida de los individuos, como son experiencias, emociones y sentimientos, se han expresado mediante símbolos (figuras rupestres, emblemas, logotipos), es así que estas formas de expresiones visuales han sido objeto de estudio para comprender su potencial de fortalecimiento del sentido de pertenencia hacia un grupo, producto u organización (Ramírez y Puerta, 2016).

En este contexto para las instituciones de educación superior, toma relevancia el sentido de pertenecía de los estudiantes, dado que contribuye a formar la imagen de la Universidad, una identificación con la marca, convirtiéndose esta en uno de los elementos para ampliar su difusión. La distinción y vinculación con la marca universitaria se relaciona con el comportamiento de compra que incide en los estudiantes a tomar la decisión de adquirir artículos promocionales y de recuerdo alusivos a su universidad; e involucra conductas como la lealtad, disposición a pagar un precio y la resistencia a aceptar información negativa sobre la universidad (Rubio, Villaseñor y Oubiña, 2015).

La adquisición de artículos oficiales universitarios por parte de los individuos afiliados directamente a la institución se ve motivada principalmente por el sentido de pertenencia. Tal es el caso de vestir una prenda con el símbolo de la universidad para acentuar su orgullo e integración en la comunidad universitaria, por medio de la compra de artículos promocionales que continuamente muestran a las demás personas (Phillips, Roundtree y Kim, 2014). Los espacios destinados a la comercialización de artículos de souvenirs universitarios son áreas de interacción de la marca con sus diferentes públicos. Las tiendas universitarias tienen como misión el fomentar la identidad universitaria, ya que, al presentar la marca de manera dinámica, aviva el sentido de pertenencia de sus comunidades estudiantiles, egresados y empleados (Universidad Pontificia Bolivariana, 2018). Los resultados de los estudios de Ramírez y Puerta (2016) plasman que el principal motor que incentiva el adquirir artículos de souvenirs es el sentido de pertenencia de los alumnos, así como también el sentimiento de orgullo hacia el recinto educativo, el cual crece al momento de portar y lucir los artículos alusivos de su universidad.

\section{Antecedentes}

Los estudios sobre el sentimiento de pertenencia en el ámbito educativo han sido abordados en años recientes en comparación de otros fenómenos sociales. Entre los pioneros se encuentra Finn (1989) quien expresa que el grado de los estudiantes en comprometerse con la escuela se basa especialmente en un elemento emocional denominado "identidad con la escuela". Por otro lado, la identidad, es un vocablo que engloba aspectos sociales, psicológicos, etc., es decir, las personas hacen frente a una diversidad de situaciones que incurren directamente en la manera que se perciben en diferentes esferas de actuación. Las personas, a través de su vida, forjan y preservan un pasado que los identifique individualmente, su moral e intelecto se adquiere en los ambientes donde ha sentido ser parte de ellos y en los que logra desenvolverse (Weil, 2002). Para que la persona conozca su identidad, es necesario indague en uno mismo ante la carencia de su autoconocimiento propio para así aceptarse y pueda descubrir lo que anhela y con ello enfoque sus esfuerzos en conseguirlo dentro del marco de normas y valores dictados en la sociedad (Frank, 1977, citado por Valdez et al., 2011). La esencia del ser humano es la de un ser social que se 
relaciona con otros semejantes que le colaboran en crear fenómenos sociales que lo benefician, pero es imposible negar que es un ser individualista, sólido y creativo. La socialización se suscita dentro de un grupo u organización social en donde acontece este proceso, esta agrupación es el eje principal de la interacción e influye en el desarrollo de la personalidad, con la cual la persona ha ido evolucionando y adaptando, inconscientemente, su actuar social a través de la experiencia (Velázquez, 2008).

En este contexto, se hace presente la memoria colectiva, cuya concepción deriva del consentimiento propio de las evocaciones de vivencias ya ocurridas, comunicadas e intercambiadas entre los pertenecientes de uno o varios grupos al interactuar y que estas son relevantes para el presente y colaboran a la permanencia de la entidad social (Cappello, 2015). El desarrollo de esta memoria colectiva, favorece al progreso de un sentido de permanencia con peculiaridades similares que conforman la identidad colectiva; encausando a las personas a comparar, reconocer y compartir colectivamente un pasado semejante, un presente valorado y un futuro alentador. Para Imaz (2015) la identidad colectiva se relaciona con el éxito que puede alcanzar una organización que desee abrirse al mercado; es así que la presencia de una imagen e identidad no fuera posible si las empresas no perfilan una marca que transmita su identidad al consumidor y que esta logre desarrollar una imagen con la cual posicionarse en el mercado por encima de otras.

Respecto a marca sus orígenes se ubican en la antigua Grecia y Roma, donde los artículos eran señalizados por símbolos que le adjudicaban carácter comercial (Davis, 2002), la finalidad era mostrar lo que almacenaban los contenedores, era común ilustraciones de uvas en botellas de vino (Moor, 2007). Estipular si el producto fue elaborado por un fabricante (propiedad) o si provenía de una región (denominación de origen), fueron los primeros pasos de cómo se concebía la identidad de marca y su comunicación (Atkin, 2008). Durante el siglo XXI, los estadounidenses se adentraron en la cuestión visual y diferenciaron la marca y producto como elementos individuales, ya que era común emplearlos de manera similar (Pastor, 2003). La marca cobró vida con el propósito de diseñar publicidad estratégica, esta tendencia fue respaldada en los diseños de identidad corporativa, la cual es un reflejo visual de las transformaciones administrativas, de la gestión operativa, de la historia, filosofía, de la personalidad, así como de su reglamentación (Ind, 1992).

Diversos factores externos inciden en la identidad de marca, lo cual puede derivar en una comunicación inexacta de su imagen. La intención de las empresas es que su identidad no se vea mermada por el entorno que la rodea el cual se ve afectado por situaciones inmersas en él como costumbres, razonamiento y comportamiento, y es precisamente el modo de actuar lo que provoca caer en influencias conductuales a los seres humanos, es decir, tienden imitar hábitos ligados a la identidad del grupo social primario al cual se forma parte (Tomasello, 2013). Keller (2013) afirma que en el transcurso del desarrollo de la identidad es crucial señalar las afiliaciones fuertes con la marca. Es necesario fijar una idea concisa de qué es, a quién va dirigida y cuál es su finalidad, lo que se traduce en los puntos ejes de la filosofía de cualquier empresa: misión, visión y valores. Al no plasmarlas con sinceridad, será complicado difundir una identidad fija y perpetua de la marca, lo que conllevará a una ausencia de valor, con escasa estabilidad en el mercado y carente de asociación por parte de los consumidores.

\section{Identidad y pertenencia}

La identidad se conceptualiza como dinámica y progresiva de acuerdo con las interacciones en las que 
están inmersos como sujetos pertenecientes a un contexto determinado (Sayago, Chacón y Rojas, 2008). Para Dubar (1991), la identidad es la derivación ininterrumpida y eventual, individual y grupal, subjetiva y objetiva, histórica y ordenada de los diversos procesos en que las personas se relacionan y que, de forma paralela, van construyendo los individuos y las sociedades. Sería imposible alcanzar una integra conciencia de uno mismo si se excluye la propia pertenencia grupal, el afecto a estos grupos y el peso que estos poseen sobre las creencias, percepciones y conductas (Gómez, Gaviria y Fernández, 2006). La manera de cómo un individuo se concibe a sí mismo se deriva de su idea de pertenencia a una o varias agrupaciones sociales y esto va ligado con el grado de valor emocional que se le otorga por formar parte de dichos grupos (Morley, 1982).

Por otro lado, la pertenencia se expresa como el sentimiento de integración personal en un colectivo social, de manera que el individuo cree y esta consiente que su permanencia en él es vital e integral (Anant, 1966, citado por Dávila y Jiménez, 2014); además no hay que perder de vista que, la pertenencia es una necesidad innatas del hombre propuesta en la pirámide jerárquica de necesidades básicas humanas (Maslow, 2012). El sentido de pertenencia es una fase de cómo ir construyendo la identidad social (Harris y Cameron, 2005), es una acumulación de sentimientos que tienen su origen de las actividades del día a día en espacios cotidianos; el nivel de este sentido de pertenencia oscila con el tiempo en la medida que las experiencias aumentan y se fortalece con el entendimiento de un espacio (Fenster, 2004).

Un entorno donde se suscitan experiencias de progreso y madurez son los recintos educativos, en los cuales se invierte gran parte de tiempo de la vida, los profesionistas que egresen de ellos llevarán siempre el nombre de su institución (Zorrilla, 2010). El sentido de pertenencia universitario es el sentimiento de orgullo de su institución, de sentirse parte de ella, estableciendo conexiones entrañables con sus integrantes; es la inclinación y aprobación de normas escolares, así como la aceptación de la valoración a la educación (Finn, 1989). Diferentes investigaciones (Kenney, Dumont y Kenney, 2005; Boyer, 1990; Astin, 1984) asientan que el estudiante al verse y percibirse como miembro, da sus primeras demostraciones de interés en lo referente a asuntos afines con la institución y colabora de forma personal con la imagen de su universidad, generando así un desarrollo educativo fluido y efectivo.

El entorno universitario es aquel lugar en el cual el alumno expresa y comparte sus sentidos con sus colegas, resultando crucial para el desarrollo de su identidad. A si, el espacio universitario fija un sistema de acuerdos y pautas para las relaciones de convivencia (Molina, 2015). La identidad universitaria, puede ser definida como el conjunto de componentes, perfiles y condiciones propias de una institución de nivel superior, que la distinguen de forma singular, le otorgan peculiaridad, y que se proyectan por medio de insignias que representan los valores que aspiran los estudiantes a alcanzarlos como propios (Linarez, 2006). Para Cabral y Villanueva (2006) es "la identificación a una colectividad institucionalizada, según las representaciones que los individuos se hacen de la realidad social y en donde se alinean factores tales como la experiencia escolar pasada, las relaciones humanas, la percepción de la universidad como unidad, el territorio, las afinidades, la educación, el vínculo, las normas y la dificultad del ingreso a la institución”.

Fomentar la pertenencia universitaria, demanda de la difusión y colaboración de un programa de identidad que muestre a la institución misma, basado especialmente en su filosofía y símbolos, con el objetivo 
que sus miembros lleguen a sentirse integrantes de una misma comunidad universitaria (Islas, 2014). Los símbolos universitarios están presentes en la vida académica de todo estudiante, se cuenta con ellos desde el momento de integrarse como alumnos a la institución, ayudando y facilitando a distinguirlos como universitarios (Castañeda, 2009). Por medio de un emblema o símbolo, toda institución educativa trata de diferenciarse de las demás, buscando mostrar su identidad dentro y fuera de sus entornos para así ir fijando su lugar en la sociedad (De Jesús, Urzúa y Vargas, 2013).

La marca es percibida e interpretada dentro de una empresa con sus integrantes, ellos pueden asumir como un compromiso el cumplimiento de la misión y visión, creando así un lazo emocional con la empresa (Estrella, 2005). Para Ramírez y Gochicoa (2010) la identidad corporativa es "el conjunto de símbolos, comunicación y comportamiento de una empresa, basados en la visión y misión de la misma, es decir, constituye la personalidad de la empresa, la identidad deseada y la identidad real”.

La mezcla de identidad (Birkigt y Stadler, 1986) plantea la combinación de tres recursos (simbolismo, comportamiento y comunicación) que una empresa utiliza para expresar cómo es en realidad su identidad y permite presentar a su mercado objetivo la imagen que se desea reflejar. El simbolismo es toda expresión visual que influyen en la forma en cómo se ve la empresa (Melewar y Jenkins, 2002), es el sistema de identidad corporativa (logotipo, colores), estética corporativa (arquitectura, diseño) y uniformidad del personal (vestuario). El comportamiento es la parte no verbal (intangible), son las gestiones conductuales hacia sus empleados y clientes; y la comunicación es la gestión de herramientas verbales y visuales (internas y externas) para transmitir un mensaje harmónico, eficaz y eficiente al grupo objetivo, generando así relaciones estables (Pérez y Rodríguez, 2014).
Las compañías cuentan con características únicas y visualmente reconocibles que ayudan a proyectar su identidad y que al impulsarlas ayudan a desarrollar su imagen visual. La identidad visual es "la traducción simbólica de la identidad de una organización” (Pintado y Sánchez, 2013), son los elementos visuales que forman parte de la marca, entre ellas distintas aplicaciones específicas como la tipografía y los colores (González, 2002, citado por Subiela, 2017). La identidad visual está compuesta por numerosos bienes tangibles, propiedad de la compañía (inmuebles, puntos de venta, vehículos, artículos, etc.), que al estamparles su marca se desempeñan como promotores de su identidad (Schmitt y Simonson, 1998). Es la pieza más importante de la identidad corporativa, ya que avala a la marca para asegurar solidez, coherencia y sinergia (Subiela, 2017). De acuerdo con Villafañe (2016) es un instrumento para mejorar la imagen pública empresarial, ya que refleja su identidad y además tiene la característica de adaptarse con el tiempo a los cambios y requerimientos a través de los años, lo que demanda una mayor normatividad para su aplicación.

En las instituciones educativas de nivel superior concurren problemas que repercuten en el proceso de generar identidad. El crecimiento intensivo del número de alumnos en las universidades, sobre todo en instituciones públicas, ha provocado una identidad universitaria confusa y poco notoria en su comunidad estudiantil (Sammartino, 2000, citado por, Molina, 2015). Este mismo fenómeno también se presenta en instituciones privadas donde los resultado reflejan que más de tres cuartas partes de los estudiantes poseen niveles de identidad universitaria regulares a bajos (Guerrero, 2017).

Los estudios llevadas a cabo por Lobo (2016) en la Universidad Peruana Unión, demuestran que el nivel de identidad universitaria de los alumnos disminuye con el paso de los años escolares debido a que las actuales 
sociedades estudiantiles (y probablemente las generaciones por venir) se encuentran necesitada de novedosos procesos que ayuden a estimular y sentir la motivación e identificación hacia algo. La investigación de Ramírez y Puerta (2016) realizada en la Universidad Nacional de Colombia, señala que el principal motor que estimula la adquisición de artículos de souvenirs es el sentido de pertenencia de los alumnos, además que el hecho de comprarlos engrandece el sentimiento de orgullo hacia la institución al poder exhibir artículos alusivos de la universidad. A pesar que diversas investigaciones y estudios resaltan la importancia del sentir y modo de actuar del miembro universitario, las cuales fomentan la identidad universitaria y el sentido de pertenencia; estas aún tienen camino que recorrer para ser asociadas como puntos clave a evaluar por parte de la mayoría de las instituciones de educación superior, ya que en muchas de ellas no se presenta normatividad institucional que gestione e impulse estos sentimientos dentro de su comunidad universitaria, y de la misma manera tampoco se pautan guías o índices que ayuden a evaluar y medir estas tendencias a través de los años.

La Universidad de Sonora realiza periódicamente estudios enfocados a alumnos, egresados y empleadores, con el propósito de evaluar la pertinencia de su oferta educativa, y su contribución en el crecimiento y desarrollo de la misma, así como el de su comunidad (Dirección de Planeación Unison, 2020) . Dichos estudios abordan temas como, calidad de los programas educativos, planta docente, la calidad de los servicios y la utilidad de su formación para desarrollarse en el ámbito laboral, entre otros. Sin embargo, se puede profundizar también en aspectos sobre nivel de pertenencia de la comunidad académica y estudiantil referente a su sentido de pertenencia e identidad Institucional.

Esta investigación tiene el propósito de conocer el sentido de identidad y pertenencia de la comunidad estudiantil universitaria hacia la institución, así como el reconocimiento de su filosofía, antecedentes y cultura general universitaria; en este caso de los alumnos de licenciatura de la Universidad de Sonora, campus Hermosillo, dentro de la División de Ciencias Económicas Administrativas, que se encuentren cursando sus últimos dos semestres. Además, esta investigación aborda una evaluación hacia el conjunto de atributos inherentes de la universidad, siendo estos los elementos gráficos que dan imagen, presencia y distinción, es decir, cómo perciben al conjunto de elementos tangibles e intangibles que conforman la identidad visual. Además, se identifica el comportamiento hacía la adquisición de artículos universitarios (souvenirs), frecuencia de compra, lugar de adquisición, es decir, una noción del posicionamiento del souvenir.

El punto inicial del estudio es el contexto en el que se encuentran inmersos los souvenirs en relación a su mercado estudiantil. La Universidad de Sonora cuenta con puntos de venta de artículos promocionales. Uno de ellos es la Librería Unison, la cual complementa su venta principal con souvenirs, artículos con los símbolos representativos y alusivos al alma mater. Esta unidad de negocio tiene presencia en tres de sus seis campus, y cuenta con cuatro sucursales (Librería Unison, 2020). Durante el periodo de 2015-2019 la tendencia de participación de compra del mercado estudiantil en las librerías universitarias, deja entrever un escenario escaso de interés de los alumnos por adquirir productos de souvenirs. En los últimos cinco años esta participación ha experimentado fluctuaciones menores de periodo en periodo, alcanzando un promedio de $0.65 \%$ por ciclo escolar (Tabla 1). Se observa que en los ciclos escolares que coinciden con nuevo ingreso se presentan incremento en los volúmenes de ventas (Librería 
Unison, 2020). La serie histórica de la matrícula estudiantil, de nivel licenciatura tiene un aumento del $12.07 \%$ en los últimos cinco años, pasando de un total de 28,309 a 31,727 entre periodos 2015-2 a 2019-2 (Dirección de Planeación Unison, 2020).

\section{Tabla 1}

Participación estudiantil por compra de souvenirs en librerías

\begin{tabular}{ccccccccccc}
\hline Ciclo & $2015-1$ & $2015-2$ & $2016-1$ & $2016-2$ & $2017-1$ & $2017-2$ & $2018-1$ & $2018-2$ & $2019-1$ & $2019-2$ \\
\hline $\begin{array}{c}\text { Población } \\
\text { Estudiantil }\end{array}$ & 24,852 & 28,309 & 25,085 & 28,783 & 25,410 & 28,702 & 25,264 & 29,050 & 25,904 & 31,727 \\
$\begin{array}{c}\text { Tickets de } \\
\text { Estudiantes }\end{array}$ & 115 & 282 & 144 & 337 & 127 & 211 & 64 & 90 & 124 & 335 \\
$\begin{array}{c}\text { Participación } \\
\text { del Mercado }\end{array}$ & $0.46 \%$ & $0.99 \%$ & $0.57 \%$ & $1.17 \%$ & $0.49 \%$ & $0.74 \%$ & $0.25 \%$ & $0.31 \%$ & $0.48 \%$ & $1.06 \%$ \\
\hline
\end{tabular}

Fuente: Elaboración propia con base en Librería Unison (2020) y Dirección de Planeación Unison (2020).

El porcentaje de participación del mercado estudiantil en la compra de souvenirs proviene del registro de las ventas al momento de identificarse como estudiantes vigentes. Es importante señalar que varias de estas compras pudieron haber sido realizadas en diferentes ocasiones por un mercado cautivo, es decir, que uno o más estudiantes durante un ciclo escolar efectuaron una a más compras. Con lo anterior se puede inferir que el porcentaje real de participación es aún menor.

En el periodo de cinco años (2015-2019), del total de ingresos captados por souvenirs en librerías, solamente un $26.32 \%$ es procedente de compras de los estudiantes, el $73.68 \%$ restante es derivado de las compras del público en general, que en este conjunto alrededor del
$80 \%$ provienen de las compras por parte del personal administrativo, operativo y académicos. El porcentaje de ingresos por estudiantes ha sufrido diversos cambios en el periodo de cinco años (Tabla 2), pasando de un $32.66 \%$ en 2015 a un descenso de $21.91 \%$ en 2019, teniendo su punto más bajo en 2018 con 17.65\% (Librería Unison, 2020). Cabe mencionar que el registro de las ventas solo permite identificar al tipo de cliente como: estudiante, empleado y público general. Lo que limita a crear una base de datos con perfil de cliente completo para detectar nichos de mercado potenciales, como sería: el semestre en curso, licenciatura, incidencias de compra, área adscrita del empleado, etc. 
Tabla 2

Porcentaje anual de ventas de souvenirs en librerías

\begin{tabular}{ccccccc}
\hline Periodo & 2015 & 2016 & 2017 & 2018 & 2019 & $2015-2019$ \\
Estudiante & $32.66 \%$ & $33.55 \%$ & $29.35 \%$ & $17.65 \%$ & $21.91 \%$ & $26.32 \%$ \\
General & $67.34 \%$ & $66.45 \%$ & $70.65 \%$ & $82.35 \%$ & $78.09 \%$ & $73.68 \%$ \\
Total & $100 \%$ & $100 \%$ & $100 \%$ & $100 \%$ & $100 \%$ & $100 \%$ \\
\hline
\end{tabular}

Fuente: Elaboración propia con base en Librería Unison (2020).

Estos resultados dejan entrever la baja participación de estudiantes en la adquisición de souvenirs universitarios. Es en este sentido, el presente estudio aborda el análisis de identidad universitaria, identidad visual y sentido de pertenencia que permea en los alumnos de licenciatura con la finalidad de obtener una descripción de sus apreciaciones, sentido de orgullo hacia su universidad. Lo cual permitirá contrastar los resultados de pertenencia e identidad y el comportamiento de compra que manifiesten hacia los souvenirs.

\section{Metodología}

Se emplea el modelo de enfoque cuantitativo, en el análisis estadístico y medición numérica de variables para analizar relación entre ellas que sustenten patrones de comportamiento (Lerma, 2009). El estudio es con alcance descriptivo, ya que permite describir las propiedades, características y perfiles de personas, grupos, procesos o cualquier otro objeto que se analice. Dicho estudio pretende medir información de manera independiente o conjunta de los conceptos o variables objeto de estudio (Malhotra, 2008). Es considerado en vertiente transversal, al tener la particularidad de que los datos son recabados dentro de un tiempo establecido.

Respecto a la población de estudio se determina a los estudiantes de la Universidad de Sonora de nivel licenciatura, ya que en ellos se encuentra el fenómeno situacional a estudiar. La población atiende los siguientes criterios: 1) Tiempo: ser estudiante cursando el ciclo escolar 2020-1, comprendido del mes de enero a mayo, 2) Espacio: estudiantes del campus Hermosillo de la división económico administrativo, 3) Homogeneidad: alumnos de la licenciatura de economía y mercadotecnia cursando sus últimos dos semestres. Le elección de dichos criterios obedece, por un lado, a razones históricas, la fundación de la universidad en 1942 sucede en este campus, el cual alberga la mayoría de alumnos matriculados. La división económicoadministrativo (DCEA) acoge las carreras pilares seleccionando la licenciatura de economía al ser una de las más longevas desde 1960 (Universidad de Sonora, 2020); y, por otro lado, razones sociales, se elige la licenciatura de mercadotecnia por ser de reciente creación que cuenta con varias generaciones egresadas. Se busca un equilibrio entre una licenciatura de inicios de la institución y de reciente oferta; se elige a los alumnos que cursan sus últimos dos semestres, debido a que la identidad y la pertenencia llevan un proceso de desarrollo en los individuos, el cual se forja a través del tiempo y conforme se viven experiencias.

Para determinar la población, se toma como referencia los planes de estudios de ambas licenciaturas para así 
identificar la cantidad de alumnos que al inicio del ciclo 2020-1 cumplen con la acumulación de créditos aprobados para ser considerados formalmente dentro de los dos últimos semestres de su licenciatura (Dirección de Servicios Escolares Unison, 2020). Los sujetos de estudio son estudiantes que cumplen con los créditos aprobados requeridos y los ubica cursando los dos últimos semestres de licenciatura, los cuales corresponde a 218 créditos en economía y en mercadotecnia 234 créditos, identificándose una población de 156 alumnos (Tabla 3). La determinación de la muestra se basa en muestreo probabilístico, estratificado (Hernández, Fernández y Baptista, 2014). El muestreo estratificado proporcional está conformado de 43 alumnos (27.6\%) de licenciatura en economía y 113 alumnos (72.4\%) en mercadotecnia. Para el cálculo de la muestra se aplica la fórmula para poblaciones finitas, con un nivel de confianza de 95\%, margen de error de $5 \%$, y un 50\% de probabilidad que ocurra o no ocurra. El resultado de la muestra fue de 112 alumnos, de los cuales el $27.6 \%$ corresponde a economía y el $72.4 \%$ a mercadotecnia.

\section{Tabla 3}

Alumnos de economía y mercadotecnia en campus Hermosillo por créditos acumulados, ciclo 2020-1

\begin{tabular}{cccc}
\hline Lic. Economía & \multicolumn{2}{c}{ Lic. Mercadotecnia } \\
\hline Créditos Acumulados & Alumnos & Créditos Acumulados & Alumnos \\
Semestre inferiores $(<218)$ & 106 & Semestre inferiores $(<234)$ & 407 \\
Dos últimos semestres $(\geq 218)$ & 43 & Dos últimos semestres $(\geq 234)$ & 113 \\
\hline
\end{tabular}

Fuente: Dirección de Servicios Escolares Unison (2020).

Es importante mencionar la delimitación de la muestra de estudio, implica que el hallazgo solo representará a una parte de la población de estudio, por lo que esta investigación, puede servir como punto de soporte para la continuidad del estudio en periodos posteriores, ampliando a demás licenciaturas.
El cuestionario es el instrumento utilizado para la obtención de datos, el cual está conformada principalmente por variables (Figura 1), las cuales a su vez están divididas en secciones llamadas planos para un mejor análisis puntual. 


\section{Figura 1}

Estructura de análisis de las variables

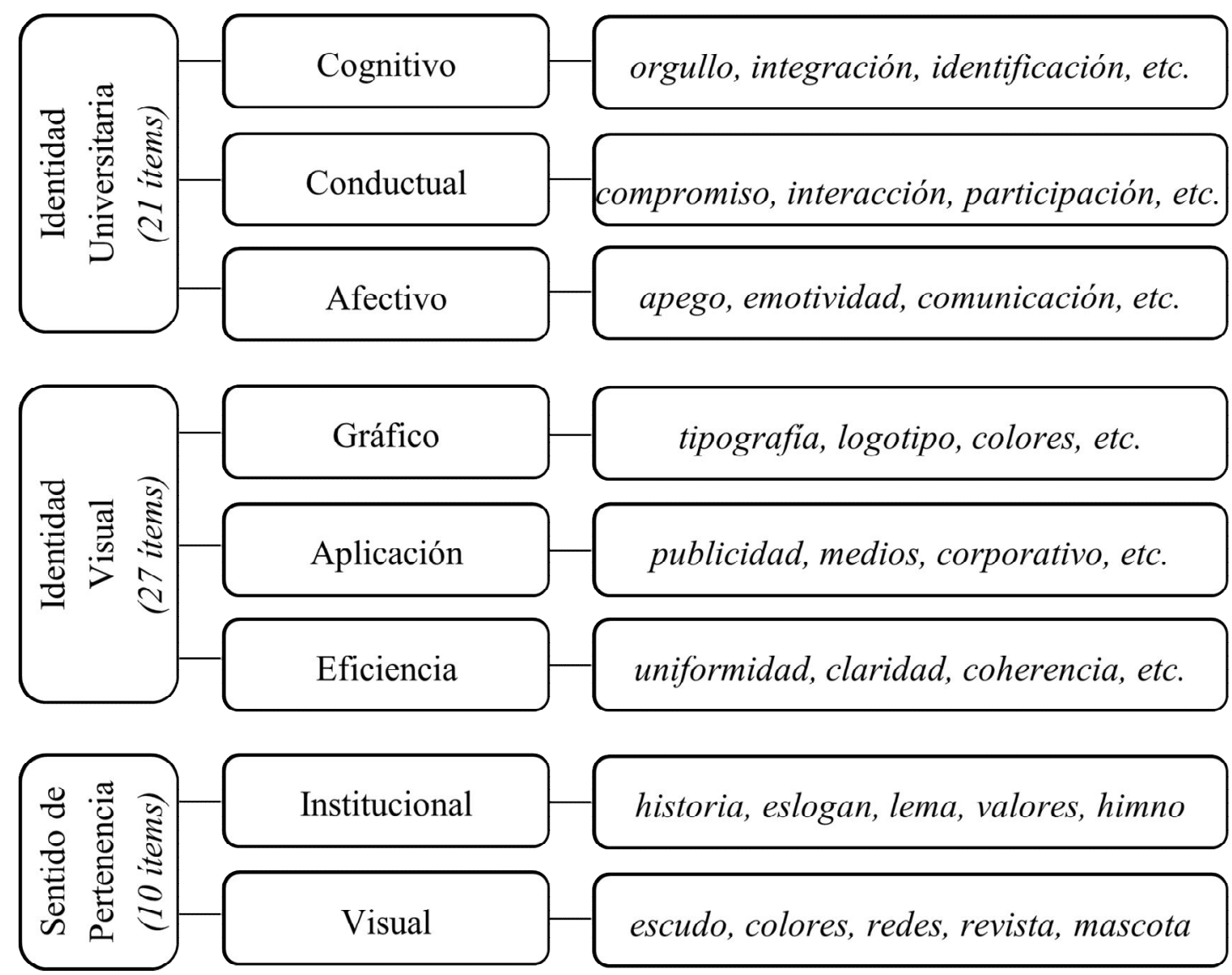

Fuente: Elaboración propia con base en Lobo (2016).

Variable 1: identidad universitaria. Se basa en el cuestionario "TPII: Test de Percepción de Identificación Institucional” (Lobo, 2016), el cual se inspira del instrumento "Prueba parcial del modelo reformulado de identificación organizacional” (Mael y Ashforth, 1992); $\mathrm{y}$ se desglosa en tres secciones llamadas planos (cognitivo, conductual y afectivo), que describen la opinión de los estudiantes a distintas situaciones, escenarios y aspectos del día a día dentro de la universidad. Al constar con 21 ítems, la puntuación mínima a obtener es de 21 y la máxima posible de 105, quedando la escala de intervalo de evaluación de baremo en: Deficiente $=21$ a 48, Regular $=49$ a 76, Buena $=77$ a 105.

Variable 2: identidad visual. Se basa en el "TPIV: Test de Percepción de Identidad Visual” (Lobo, 2016) y se divide en tres planos (gráfico, aplicación y eficiencia), los cuales reflejan como los estudiantes perciben los elementos tangibles e intangibles que dan imagen y 
presencia a la institución. Al contar con 27 ítems, la puntuación mínima es de 27 y la máxima de 135, su escala de baremo es: Deficiente $=27$ a 62, Regular $=63$ a 98, Buena = 99 a 135 .

Variable 3: sentido de pertenencia, es un examen de conocimiento general universitario, donde se hacen afirmaciones con la finalidad de reflejar si los alumnos pueden detectar la veracidad de ellas, con respuestas dicotómicas sí/no. Se divide en dos planos (institucional y visual). Compuesta de 10 ítems, la puntuación mínima a obtener es de 0 puntos y la máxima posible es de 10 puntos, su escala de baremo es: Deficiente $=0$ a 3.2, Moderada $=3.3$ a 6.5, Buena $=6.6$ a 10 .

La evaluación de los planos es a través de la escala de baremo, las clases de sus intervalos presentan valor categórico deficiente, moderada y buena, la variación de sus rangos de puntuación es según la cantidad de ítems que esté compuesto el plano y la puntuación proviene, para variable 1 y 2, del puntaje obtenido al utilizar la escala de Likert con valores de 1 a 5 puntos para asignar opinión de aprobación y la variable 3 proviene de los aciertos al responder opciones dicotómicas.

Variable 4: posicionamiento del souvenir e interés de identidad, tiene como finalidad el poder identificar los comportamientos del mercado estudiantil hacia el producto souvenirs universitario, ya que este ayuda a promover la identidad universitaria al proyectarla de forma dinámica y estimula el sentido de pertenencia. Para finalizar, se cuestiona el interés en conocer más acerca de la historia e identidad de la universidad.

\section{Resultados}

En lo que se refiere a las características demográficas correspondiente a la muestra se observan las siguientes proporciones, en donde el $45.5 \%$ son del género masculino y $54.5 \%$ del género femenino; en lo que respecta al programa académico al cual se encuentra inscrito se tiene que el $72.4 \%$ de los estudiantes corresponden al programa de la licenciatura en mercadotecnia y el $27.6 \%$ al programa de la licenciatura en economía; por otro lado $55.35 \%$ de los estudiantes expresaron estar laborando, mientras el 44.65\% únicamente se dedica al estudio, el $100 \%$ de los encuestados su estado civil es soltero.

Se observa que el $60 \%$ de los estudiantes indica un grado de identidad universitaria moderada y el $40 \%$ en el rango de nivel de identidad buena. Dentro del rango de identidad moderada se concentra el 53\% de las mujeres y el $63 \%$ de los hombres. Ninguno se concentra dentro de la evaluación deficiente.

Respecto al plano cognitivo, refleja la integración de los estudiantes a la universidad, si se sienten identificados con las actividades académicas y sociales que realiza la institución, si sus intereses y metas se complementan con las de la universidad y si al hablar de ella la refieren con satisfacción de formar parte de la misma. Las cifras de este plano reflejan un grado de integración alto, un $67 \%$ posee un resultado cognitivo bueno, mientras que el $32.1 \%$ fue con nivel moderado y en menor proporción con nivel deficiente $(0.9 \%)$. Por otro lado, el plano conductual, refleja la interacción y participación de los estudiantes en las actividades sociales que lleva a cabo la universidad, si fueron satisfactorias y de grata experiencia, cómo describen su relación con los compañeros, su compromiso con los objetivos de la universidad. Estos porcentajes reflejan un grado de interacción aceptable, el $51 \%$ posee un resultado moderado, respecto al grado de participación y convivencia con la comunidad universitaria es buena con un $49 \%$.

Por último, en el plano afectivo, se refleja el apego que los estudiantes sienten con la universidad, la emotividad hacia las metas y reconocimientos que logra la misma, los lazos de comunicación entre compañeros, 
la voluntad de apoyarlos a ellos y a la propia universidad, así como la libertad de expresión con otros miembros. Los resultados indican un grado de apego aceptable, el $62.5 \%$ de los estudiantes considera como moderado su nivel de afecto hacia la institución, un $34.8 \%$ asegura que tiene un nivel bueno, en contra parte un $2.7 \%$ admite tener un nivel deficiente.

Tanto en el plano conductual como el plano afectivo logran promedios con evaluaciones moderadas, el plano cognitivo está muy cerca del límite inferior de su rango de evaluación "buena"; la sumatoria de los puntajes promedios de cada plano otorga un resultado de 75.55 , localizando a esta media dentro del rango moderado para toda la variable. Existe un nivel medio de identidad universitaria (Tabla 4) donde sus experiencias de interrelación con la universidad y sus miembros son moderadas.

\section{Tabla 4}

Identidad iniversitaria - evaluacion por planos

\begin{tabular}{cccc}
\hline Plano (ítems) & Puntaje & Rango Puntaje & Evaluación \\
\hline Cognitiva (6) & 22.62 & $22-30$ & Buena \\
Conductual (7) & 25.39 & $16.3-25.5$ & Moderada \\
Afectivo (8) & 27.54 & $18.6-29.2$ & Moderada \\
General (21) & $\mathbf{\Sigma}=\mathbf{7 5 . 5 5}$ & $\mathbf{4 9 - 7 6}$ & Moderada \\
\hline
\end{tabular}

Fuente: Elaboración propia con base en resultados de investigación.

En cuanto a la identidad visual, el $23 \%$ de los estudiantes tiene evaluación moderada, el 77\% otorga un alto grado con una evaluación buena y en esta se concentra el $77 \%$ de las mujeres y $76 \%$ de los hombres. Desde el indicador gráfico, se evalúa si los componentes gráficos de la universidad son reconocibles; esto es si el logotipo oficial es identificable y diferenciable de otras instituciones, si su estilo, colores, tipografía y elementos son adecuados y agradables. Estos porcentajes reflejan un grado de identificación muy alto, el 93\%, expresa que los elementos gráficos de la universidad son altamente reconocibles, otorgando una evaluación buena, junto con un $7 \%$ que expresa que es moderada. Referente a la aplicación, evalúa si la universidad realiza una gestión adecuada de su imagen externa, si sus campañas publicitarias se distinguen de otras instituciones, gestión de sus medios digitales y de su infraestructura. En este sentido el 58\% evalúa con nivel bueno la universidad cumple con una gestión de su imagen, un 39.3\% asegura que lo cumple de forma moderada, y tan sólo un $2.7 \%$ admite la universidad lo implementa con nivel deficiente. En lo que concierne al plano de eficiencia, se mide la efectividad de los materiales publicitarios, si estos son considerados llamativos y agradables a la vista, si presenta igualdad y semejanza de estilo, si los mensajes que transmiten son fáciles de entender y congruentes con la idea que exponen. Al respecto un $74.1 \%$ otorga un nivel de 
eficiencia bueno al contenido de la publicidad, un 23.2\% expresa que tiene una eficiencia moderada y en contra parte, el 2.7\%, la evalúa como deficiente.

El plano gráfico como el plano de eficiencia logran promedios con evaluaciones buenas, el plano aplicación está casi al límite superior de su rango moderado. La sumatoria de los puntajes promedios otorga un resultado de 106.48 (Tabla 5), localizando a esta media dentro del rango bueno para la variable de identidad visual.

\section{Tabla 5}

Identidad visual - evaluación por planos.

\begin{tabular}{cccc}
\hline Plano (ítems) & Puntaje & Rango Puntaje & Evaluación \\
\hline Gráfico (9) & 39.41 & $33-45$ & Buena \\
Aplicación (10) & 36.48 & $23.3-36.5$ & Moderada \\
Eficiencia (8) & 30.59 & $29.3-40.0$ & Buena \\
General (27) & $\mathbf{\Sigma}=\mathbf{1 0 6 . 4 8}$ & $\mathbf{9 9 - 1 3 5}$ & Buena \\
\hline
\end{tabular}

Fuente: Elaboración propia con base en resultados de investigación.

Acerca del sentido de pertenencia, el 73.2\%, obtuvieron un resultado medio con una evaluación moderada sobre la cultura universitaria, mientras un $18.8 \%$ posee con un grado de conocimiento alto. Con un sentido de pertenencia moderada la proporción de las mujeres (77\%) es ligeramente mayor que de los hombres (68.6\%). Para el plano institucional, enfocado a aspectos de la filosofía y antecedentes universitarios; los resultados reflejan un alto desconocimiento en aspectos como: himno, historia y valores, con 57\%, 72\% y 76\% respectivamente; en cuanto al eslogan el $83 \%$ si lo identifican, así como al lema con el 51\%. El análisis general refleja que el $70.54 \%$ se concentra en los rangos moderado. Respecto al aspecto visual, encausado a conocer si los estudiantes detectan aspectos simbólicos y comunicativos de su universidad, destaca un alto reconocimiento en aspectos como: colores, revista oficial y mascota, con 70\%, 70\% y 72\% respectivamente, así como a las redes sociales con $61 \%$; mientras el $58 \%$ tiene dificultadas para identificar los elementos del escudo. El análisis general del plano refleja que el $95.5 \%$ de la muestra se concentra en los rangos moderado y bueno.

El plano institucional y el visual logran promedios similares de 2.29 y 3.14 cada uno, con evaluaciones moderadas. El resultado general de la variable es de 5.43 (Tabla 6), ubicándose en el rango moderado. 


\section{Tabla 6}

Sentido de pertenencia - evaluación por planos

\begin{tabular}{cccc}
\hline Plano (ítems) & Puntaje & Rango Puntaje & Evaluación \\
\hline Institucional (5) & 2.29 & $1.6-3.2$ & Moderada \\
Visual (5) & 3.14 & $1.6-3.2$ & Moderada \\
General (10) & $\mathbf{\Sigma}=\mathbf{5 . 4 3}$ & $\mathbf{3 . 3}-\mathbf{6 . 5}$ & Moderada \\
\hline
\end{tabular}

Fuente: Elaboración propia con base en resultados de investigación.

Al analizar el posicionamiento del souvenir e interés de identidad, un 61\% de los alumnos reconoce haber usado o portado en alguna ocasión un souvenir universitario. De la totalidad de los encuestados, $82 \%$ está consciente y tiene noción de una tienda universitaria que comercialice souvenirs; y tan solo un $18 \%$ de los alumnos desconoce su existencia. Por otro lado, un $62.5 \%$ de estudiantes reconoce nunca haber realizado la compra de un souvenir, mientras un $37.5 \%$ admite haber comprado un artículo promocional. De los estudiantes que compran, el $44 \%$ corresponde a mujeres y el $29 \%$ a hombres. El lugar preferente de compra de souvenirs son las Librerías Unison, ya que sobresalen como el lugar de compra con el 71\% de las menciones. En lo que concierne a la historia e identidad de la universidad, el $56 \%$ expresó falta de interés y un $44 \%$ sí desea aprender sobre estos aspectos.

\section{Conclusiones}

Los estudiantes de la Universidad de Sonora en la División de Ciencias Económico Administrativas cuentan con un nivel moderado respecto a la variable Identidad Universitaria, en cuanto a temas de filosofía, antecedentes y cultura universitaria; identidad corporativa, así como en aspectos simbólicos y comunicativos, Sin embargo, en este rubro destaca con un nivel bueno por parte de los estudiantes aspectos de sentirse identificados con las actividades académicas y sociales de la institución y satisfacción de formar parte de la misma. Respecto a la variable identidad visual de la Universidad, alcanza evaluaciones altas, lo que implica los estudiantes perciben los elementos tangibles e intangibles que dan imagen y presencia a la institución. Sobresales un alto reconocimiento alto en simbología universitaria, los estudiantes expresan que los elementos gráficos de la universidad son muy reconocidos. En cuanto a la publicidad universitaria y sus mensajes, son detectados y entendidos fácilmente al presentar coherencia y balance, así como que su contenido visual en los medios impresos y digitales lo perciben con uniformidad de estilo. Acerca de la variable de pertenencia la evaluación fue moderada, en temas como himno, historia y valores los resultados muestran un alto desconocimiento. En cuanto al plano visual: colores, mascota, redes sociales tienen alto reconocimiento. En cuanto al posicionamiento de los artículos promocionales "souvenirs", un alto porcentaje identifica las tiendas universitarias de comercialización estos artículos, pero el 62\% manifestó no haber comprado un artículo promocional.

Los resultados dejan entrever que la variable de identidad visual tiene mayor reconocimiento en los 
estudiantes, lo cual contribuyen en una valoración alta de imagen de marca "UNISON", aunando a los valores altos de evaluación del plano cognitivo de la identidad que expresa satisfacción de ser parte de la institución, se observa el estudiantado percibe los elementos que la conforman, ayudando así a transmitir una identidad fija y duradera de la marca al otorgarle una posición estable. Sin embargo, los estudiantes presentan una baja asociación, como mercado de consumo, ante el producto souvenir. A pesar del incremento de ingreso de población estudiantil, aunado al porcentaje considerable de alumnos que admite haber usado algún artículo promocional alusivo a la Unison y siente orgullo de pertenecer a la institución, estos resultados, no mantienen sinergia con las ventas de artículos promocionales universitarios.

El sentido de pertenencia y el reflejo de un nivel de orgullo "moderado" del estudiantado, puede incidir en el escaso interés de compra de souvenirs en más de la mitad de los encuestados. Estos hallazgos tienen pertinencia con la investigación de Ramírez y Puerta (2016), la cual sostiene que el sentido de pertenencia es la razon principal por el cual el alumno adquiere productos de souvenirs. Por otro lado, los resultados de identidad unveristaria tienen consistencia con el estudio realizado por Lobo (2016), que sustenta que el nivel de identidad universitaria va desendiendo conforme transcurren el tiempo, de los primeros años disminuye de un nivel bueno a un nivel moderado o malo. En este caso los estudiantes de ultimo semestre muestran un nivel moderado. Los resultados describen un perfil de estudiane con alto nivel de identidad visual y moderado en identidad universitaria y pretenencia.

Fava Pedroza, P. A., Velarde Mendívil, A. T., y Salgado Beltrán, L.

\section{Referencias}

Astin, A. W. (1999). Student Involvement: A Developmental Theory for Higher Education. Journal of College Student Development, 40(5), 518-529. https://www.middlesex.mass.edu/ace/downloads/astini nv.pdf

Atkin, D. (2008). El secreto de las Marcas. Clubes para gente muy especial ( $1^{\mathrm{a}}$ ed.). Barcelona, España: Robinbook.

Birkigt, K., y Stadler, M. (1986). Corporate Identity : Grundlagen, Funktionen, Fallbeispiele ( $3^{\mathrm{a}}$ ed.). Landsberg am Lech, Alemania: Moderne Industrie.

Boyer, E. L. (1990). Campus life: in search of community. A especial report. Princeton: Princeton University Press.

https://files.eric.ed.gov/fulltext/ED320492.pdf

Cabral, M. V. (2006). Identidad estudiantil universitaria en estudiantes de licenciatura. Revista

PsicologiaCientifica.com, 8(15). http://www.psicologiacientifica.com/identidadestudiates-universitarios

Cappello, H. M. (2015). La identidad universitaria. La construcción del concepto. Revista Internacional de Ciencias Sociales y Humanidades, SOCIOTAM, 25(2), 33-53.

https://www.redalyc.org/pdf/654/65452536003.pdf

Castañeda, J. (2009). Valores y símbolos universitarios. Dirección de Identidad Universitaria de la Universidad Autonoma del Estado de México. http://web.uaemex.mx/identidad/docs/valores\%20y\%2 0simboloscastaneda.pdf 
Castro S., H. E. (2015). Identidad Universitaria. UniMex Universidad Mexicana - Investigación. http://unimex.edu.mx/Investigacion/DocInvestigacion/I dentidad_Universitaria.pdf

Dávila, C., y Jiménez, G. (2014). Sentido de pertenencia y compromiso organizacional: predicción del bienestar. Revista de Psicología, 32(2), 272-302. https://www.redalyc.org/pdf/3378/337832618004.pdf

Davis, S. M. (2002). Brand Asset Management: Driving Profitable Growth Through Your Brands ( $1^{\mathrm{a}}$ ed.). San Francisco, USA: John Wiley \& Sons.

De Jesús, A., Urzúa, J., y Vargas, J. (2013). La identidad de marca UdeG en los universitarios del CUCEA. Red Internacional de Investigadores en Competitividad, 7 , 1709-1721.

https://www.riico.net/index.php/riico/article/view/372/ 1504

Dirección de Planeación Unison. (2020). Estudios de Pertinencia.

https://planeacion.unison.mx/pertinencia.htm

Dirección de Planeación Unison. (2020). Población Estudiantil, serie histórica. Sistema de Información Estadistica (SIE).

https://planeacion.unison.mx/sie/alumnos/poblacion_hi storica.htm

Dirección de Servicios Escolares Unison. (2020). Reporte de matricula de alumnos por acumulación de creditos aprobados 2020. Hermosillo: Universidad de Sonora.

Dubar, C. (1991). La socialisation. Construction des identités sociales et profesionelles. París: Armand Colin.
Estrella, A. (2005). Una cultura empresarial basada en la marca: los desafíos del branding y las franquicias de marca interna. Capital Humano (184), 40-50. http://pdfs.wke.es/2/2/4/5/pd0000012245.pdf

Fenster, T. (2004). Gender and the City: The different Formations of Belonging. En Nelson, y Seager, $A$ Companion to Feminist Geography (1 ed., págs. 242256). Malden: Blacwell. https://www.tau.ac.il/ tobiws/gender_companion.pdf

Finn, J. (1989). Withdrawing from school. Review of Educational Research, 59(2), 117-142.

https://ed.buffalo.edu/content/dam/ed/main/docs/newsl etter/Fall09-Jeremy-Finn-Withdrawing.pdf

Gómez, Á., Gaviria, E., y Fernández, I. (2006). Psicología Social (1 ed.). Madrid, España: Sanz y Torres.

Guerrero, O. E. (2017). Identidad universitaria y rendimiento académico de los estudiantes de la escuela académico profesional de educación de la Universidad Nacional Jorge Basadre Grohmann Tacna 2016. Tesis de Maestría. Tacna, Perú: Universidad Privada de Tacna. https://repositorio.upt.edu.pe/bitstream/handle/20.500.1 2969/494/Guerrero_Vargas_Oscar.pdf?sequence=1\&is Allowed=y

Harris, G. E., y Cameron, J. E. (2005). Multiple Dimensions of Organizational Identification and Commitment as Predictors of Turnover Intentions and Psychological Well-Being. Canadian Journal of Behavioural Science, 37(3), 159-169. http://web.b.ebscohost.com/ehost/pdfviewer/pdfviewer ?vid=1\&sid=28a62494-308a-47ce-a78c55aa76941299\%40pdc-v-sessmgr03 
Hernández, R., Fernández, C., y Baptista, P. (2014). Metodología de la Investigación (6ª ed.). D.F., México: McGraw-Hill Education. http://observatorio.epacartagena.gov.co/wpcontent/uploads/2017/08/metodologia-de-lainvestigacion-sexta-edicion.compressed.pdf

Imaz S., C. E. (2015). El concepto de identidad frente a imagen de marca. Tesis de Pregrado. Madrid: Universidad Pontificia Comillas. https://repositorio.comillas.edu/xmlui/bitstream/handle/ 11531/4554/TFG000672.pdf?sequence $=1 \&$ isAllowed $=$ $\mathrm{y}$

Ind, N. (1992). La imagen corporativa. Estrategias para desarrollar programas de identidad eficaces ( $1^{\mathrm{a}} \mathrm{ed}$.). Madrid, España: Ediciones Díaz de Santos. https://books.google.com.mx/books/about/La_imagen_ corporativa.html?id=-

MLwmsABLFQC\&printsec $=$ frontcover\&source=kp_re ad_button $\&$ hl $=$ es\&redir_esc $=y \# v=$ onepage $\& q \& f=$ false

Islas, O. (2014). Comunicación, identidad y sentido de pertenencia en instituciones de educación superior. Obtenido de:. Edähi Boletín científico de ciencias sociales y humanidades del ICSHu, 2(4). https://doi.org/10.29057/icshu.v2i4.921

Keller, K. L. (2013). Strategic Brand Management: Building, Measuring, and Managing Brand Equity (4 ed.). England: Pearson.

Kenney, D. R., Dumont, R., y Kenney, G. (2005). Mission and Place: Strengthening Learning and Community through Campus Design (1 ed.). Westport: Rowman \& Littlefield Publishers.

Lerma. (2009). Metodología de la investigación: propuesta, anteproyecto y proyecto (4 ${ }^{\mathrm{a}}$ ed.). Bogotá, Colombia: Ecoe Ediciones.
Librería Unison. (2020). Reportes anuales de ventas 2015-2019. Hermosillo: Universidad de Sonora.

Librería Unison. (2020). Ubicaciones.

https://libreria.unison.mx/sucursales.php?osCsid=tvs19 cff5uvbbv88ap64tagut4

Linarez, A. (2006). La Identidad Universitaria. Toluca de Lerdo: Universidad Autónoma del Estado de México.

http://web.uaemex.mx/identidad/docs/PONENCIA_ID ENTIDAD.pdf

Lobo, S. H. (2016). Relación de la identidad visual de la Universidad Peruana Unión con la identificación institucional de sus estudiantes de pregrado sede Ñaña, Lima 2015. Tesís de pregrado. Ñaña, Perú:

Universidad Peruana Unión. https://repositorio.upeu.edu.pe/handle/20.500.12840/56 1

Mael, F., y Ashforth, B. (1992). Alumni and their alma mater: A partial test of the reformulated model of organizational identification. Journal of Organizational Behavior, 13(2), 103-123. https://doi.org/10.1002/job.4030130202

Malhotra, N. (2008). Investigación de Mercados (5 ed.). México: Pearson- Prentice Hall. http://www.elmayorportaldegerencia.com/Libros/Merc adeo/\%5BPD\%5D\%20Libros\%20\%20Investigacion\%20de\%20Mercados.pdf

Maslow, A. H. (2012). Motivacion y Personalidad. Madrid: Díaz de Santos.

Melewar, T., y Jenkins, E. (2002). Defining the corporate identity construct. Corporate Reputation Review, 5(1), 76-90. https://doi.org/10.1057/palgrave.crr.1540166 
Mertens, D. (2009). Research and evaluation in education and psychology: Integrating diversity with quantitative, qualitative, and mixed methods ( $3^{\mathrm{a}}$ ed.). Thousand Oaks, USA: Sage.

Molina, J. A. (2015). Recorrido por dos ámbitos identitarios: universidad y ciberespacio. Revista Lasallista de Investigación, 12(2), 204-214. http://www.scielo.org.co/pdf/rlsi/v12n2/v12n2a21.pdf

Moor, L. (2007). The Rise of the Brands (1 ${ }^{\mathrm{a}}$ ed.). New York, USA: Berg Publishers.

Morley, I. E. (1982). Henri Tajfel's Human Groups and Social Categories. British Journal of Social Psychology(21), 189-201. https://doi.org/10.1111/j.2044-8309.1982.tb00540.x

Osterman, K. (2000). Students'need for belonging in the school community. Review of Educational Research, 70(3), 323-367.

https://doi.org/10.3102/00346543070003323

Pastor, F. (2003). Técnico en Publicidad (1 ${ }^{\mathrm{a}}$ ed.). Madrid, España: Cultural S.A. de Ediciones.

Pérez, A., y Rodríguez, I. (2014). Identidad, imagen y reputación de la empresa: integración de propuestas teóricas para una gestión exitosa. Cuadernos de Gestión, 14(1), 97-126.

http://www.redalyc.org/articulo.oa?id=274330593005

Phillips, J., Roundtree, R., y Kim, D. (2014). Mind, body, or spirit? An exploration of customer motivations to purchase university licensed merchandise. Sport, Business and Management: An International Journal, 4(1), 71-87. https://doi.org/10.1108/SBM-08-2011-0069
Pintado, T., y Sánchez, J. (2013). Imagen Corporativa. Influencia en la gestión empresarial (2 ed.). Madrid, España: ESIC.

http://sgfm.elcorteingles.es/SGFM/dctm/MEDIA01/20 1305/16/00106524422844_1_.pdf

Puga, M. C., Peschard, J., y Castro, T. (2007). Hacia la Sociología (4 ed.). México: Pearson. http://fcaenlinea.unam.mx/anexos/1141/1141_Hacia_la _sociologia.pdf

Ramírez, C., y Puerta, K. (2016). Identificación de factores motivantes de compra en tiendas universitarias: el caso de la Universidad Nacional de Colombia- sede Medellín. Revista CEA, 2(4), 89-99. https://doi.org/10.22430/24223182.174

Ramírez, J. I., y Gochicoa, E. F. (2010). Imagen corporativa: ventaja competitiva para las organizaciones PYME. Ciencia Administrativa, 2010(1), 1-8.

https://www.uv.mx/iiesca/files/2012/10/SUMARIO201 $0-1 . p d f$

Rubio, N., Villaseñor, N., y Oubiña, J. (2015). Consumer identification with store brands. BRQ Business Research Quarterly, 18(2), 111-126. https://doi.org/10.1016/j.brq.2014.03.004

Sayago, Z. B., Chacón, M. A., y Rojas, M. E. (2008). Construcción de la identidad profesional docente en estudiantes universitarios. Educere. La Revista Venezolana de Educación, 12(42), 551-561. http://www.redalyc.org/articulo.oa?id=35614569016
Fava Pedroza, P. A., Velarde Mendívil, A. T., y Salgado Beltrán, L. 
Schmitt, B., y Simonson, A. (1998). Marketing y Estética. La gestión estratégica de la marca, la identidad y la imagen ( $1^{\text {a }}$ ed.). España: Ediciones Deusto S.A.

https://books.google.com.mx/books?id=MhDetoL8mw AC\&pg=PA24\&hl=es\&source=gbs_selected_pages\&c $\mathrm{ad}=2 \# \mathrm{v}=$ onepage $\& \mathrm{q} \& \mathrm{f}=$ false

Subiela, B. J. (2017). La gestión de la identidad visual corporativa de las universidades españolas. ESS Grafica, 5(10), 115-124.

https://revistes.uab.cat/grafica/article/view/v5-n10subiela/73-pdf-es

Tomasello, M. (2013). Los orígenes de la comunicación humana ( $1^{\mathrm{a}}$ ed.). Madrid, España: Katz Editores. https://books.google.com.mx/books/about/Los_or\%C3 \%ADgenes_de_la_comunicaci\%C3\%B3n_humana.htm l?id=JFpDwAAQBAJ\&printsec $=$ frontcover\&source $=$ kp_read_ button\&hl=es\&redir_esc $=\mathrm{y} \# \mathrm{v}=$ onepage $\& \mathrm{q} \& \mathrm{f}=$ false

Universidad de Sonora. (2020). Historia de la Universidad de Sonora.

https://www.unison.mx/historia-de-la-universidad-desonora/

Universidad Pontificia Bolivariana. (2018). Tienda Universitaria. https://www.upb.edu.co/es/vidauniversitaria/editorial-libreria/servicios/tiendauniversitaria
Valdez Z., A., Huerta F., D., y Vergara O., A. (2011). Tu identidad, tu éxito: La formación de identidades póliticas y sociales como estrategias comunicativas en las campañas electorales. Razón y Palabra(78). https://www.redalyc.org/articulo.oa?id=199524192017

Velázquez, G. (2008). Sociología de la organización (1 ed.). D.F., México: Limusa. https://books.google.com.mx/books?id=x0r9HK74n1w C\&printsec=frontcover\&hl=es\&source=gbs_ge_summ ary_r\&cad $=0 \# \mathrm{v}=$ onepage $\& \mathrm{q} \& \mathrm{f}=$ false

Villafañe G., J. (2016). Imagen Positiva: Gestión estratégica de la imagen de las empresas (1 ed.). Madrid, España: Eidiciones Pirámide. https://books.google.com.mx/books/about/Imagen_posi tiva.html?id=GHAFDAAAQBAJ\&printsec $=$ frontcover \&source $=$ kp_read_button $\& h l=e s \& r e d i r \_e s c=y \# v=$ onep age \&q\&f=false

Weil, S. (2002). The Need for Roots. Prelude to a Declarationof Duties towardsMankind (1 ed.). New York, USA: Routledge. https://www.docdroid.net/rKuInyQ/simone-weil-theneed-for-roots-prelude-to-a-declaration-of-dutiestowards-mankind.pdf\#page $=3$

Zorrilla A., S. (2010). Aspectos socioeconómicos de la problemática en México (6 ed.). D.F., México: Limusa. https://books.google.co.cr/books?id=I7JwtEE__gAC\& printsec $=$ copyright $\# \mathrm{v}=$ onepage $\& \mathrm{q} \& \mathrm{f}=$ false
Fava Pedroza, P. A., Velarde Mendívil, A. T., y Salgado Beltrán, L. 